\title{
Pacific
}

Journal of

Mathematics

\section{SKEINS AND HANDLEBODIES}

W. B. RAYMOND LICKORISH

Volume $159 \quad$ No. 2 


\title{
SKEINS AND HANDLEBODIES
}

\author{
W. B. R. LICKORISH
}

The Temperley-Lieb algebra is used to find a base for the vector space that is associated to a closed surface by the Topological Quantum Field Theory corresponding to the original Jones polynomial invariant.

1. Introduction. The Kauffman linear skein $\mathbb{S} M$ of an oriented 3manifold $M$, that has a (possibly empty) finite collection of (framed) points specified in its boundary, is defined as follows. Throughout, $A$ will be a fixed complex number later to be chosen to be a primitive $4 r$ th root of unity (though it is equally possible to work with the ring of Laurent polynomials in $A$, quotiented by the ideal generated by a cyclotomic polynomial). $\mathbb{S} M$ is the vector space of formal linear sums of isotopy classes of framed links in $M$ of disjoint simple closed curves and arcs that agree with the specification in $\partial M$, quotiented by the following relations.

(i) 次" $\left.=A \stackrel{\longleftarrow}{\curvearrowright}+A^{-1}\right)($

(ii) $L \cup U=\left(-A^{-2}-A^{2}\right) L$.

In (i) a framing on a curve is depicted by a parallel to the curve, and in (ii) $U$ is a zero-framed unknotted component in a ball disjoint from the link $L$. It is often convenient to project $M$ to some surface $F$ (for example, $S^{3}$ less two points projects to $S^{2}$, a handlebody projects to a disc-with-holes) and then $\mathbb{S} M$ is interpreted as the linear skein $\mathbb{S} F$ of link diagrams in $F$ as in [5], [7], [10], the framings being determined by parallel curves in $F$. In particular the $n$th Temperley-Lieb algebra is the Kauffman skein of the ball with two sets of $n$ points specified on its boundary. It is convenient to consider that via link diagrams in a rectangle with $n$ specified points on the left edge, $n$ points on the right edge, the product in the algebra coming from juxtaposition of the rectangles. Now, it is clear that $\mathbb{S} S^{3}=\mathbb{C}$; in fact a zero-framed link corresponds in $\mathbb{S} S^{3}$ to its Jones polynomial evaluated at $t=A^{-4}$. Suppose that $M$ is embedded in $S^{3}$, that $M^{\prime}$ is the closure of $S^{3}-M$ and that $M$ and $M^{\prime}$ have the same specified framed points in their 
common boundary. There is then a natural bilinear map

$$
\mathbb{S} M \times \mathbb{S} M^{\prime} \rightarrow \mathbb{C}
$$

induced by the operation of taking framed links $L$ and $L^{\prime}$ in $M$ and $M^{\prime}$ and regarding $L \cup L^{\prime}$ as an element of $\mathbb{S} S^{3}$. Such bilinear forms will be used in the usual way to associate to any element $X$ of $\mathbb{S} M$ an element $X^{*}$ of the dual of $\mathbb{S} M^{\prime}$. This idea was considered, for the Temperley-Lieb algebra, in some detail in [6] where it was noted that the bilinear form could become degenerate when $A$ was a root of unity.

The $\mathrm{Sl}_{q}(2, \mathbb{C})$ invariant introduced by Witten [12] for a closed 3manifold "at a certain level" (here to be interpreted as "at a 4 rth primitive root of unity $A$ "), was in [7] and [8] shown to be essentially an element of $\mathbb{S} S^{3}$ (indirectly) associated to any framed link in $S^{3}$ that produces the manifold by means of surgery. Atiyah and Witten [1] laid down the axioms for a "Topological Quantum Field Theory" within the framework of which it might be desirable to view quantum invariants. That involves the association of a vector space with every closed oriented surface and an element of that space to every oriented 3-manifold bounded by the surface. Vogel [11], paying due regard to framings of manifolds, explained how the relevant Topological Quantum Field Theory for the $\operatorname{Sl}_{q}(2, \mathbb{C})$ invariant could be viewed in the context of the combinatorial methods of [7] and [8]. If a surface is regarded as the common boundary of a 3-manifold $M$ in $S^{3}$, and the closure of its complement $M^{\prime}$ as already considered, then the vector space to be associated to that surface is the quotient of $\mathbb{S} M$ by the kernel of the map $X \mapsto X^{*}$. The purpose of this paper is briefly to give a base for that quotient space, thus in principle determining its dimension, and thus giving, from this viewpoint (as explained in [1]), an approach to the $\operatorname{Sl}_{q}(2, \mathbb{C})$-invariant of the product of a closed surface and a circle.

2. Using the Temperley-Lieb algebra. In what follows diagrams represent elements of the (Kauffman) linear skein of link diagrams in a rectangle with $n$ specified points on the left edge and $n^{\prime}$ on the right edge. An integer $i$ beside an arc will denote the intended presence of $i$ parallel copies of that arc. When $n=n^{\prime}$ this skein is the TemperleyLieb algebra $T L_{n}$. Recall (for example from [7] or [8]) that this is generated, as an algebra, by elements $1, e_{1}, e_{2}, \ldots, e_{n-1}$ where 1 is $n$ arcs going straight from the left side of the rectangle to the right, and that $e_{i}$ is the same except that the $i$ th arc doubles back to the 
$(i+1)$ th point on the left edge, the $i$ th and $(i+1)$ th points on the right edge being connected similarly. For generic values of $A, T L_{n}$ contains a central idempotent $f^{(n)}$ with the properties that $f^{(n)} e_{i}=0$ for all $i, f^{(n)} f^{(n)}=f^{(n)}$ and $1-f^{(n)}$ belongs to, and is indeed the identity of, the ideal generated by $\left\{e_{1}, e_{2}, \ldots, e_{n-1}\right\}$. In the diagrams that follow a small square will denote the presence of an $f^{(n)}$ the relevant value of $n$ being deducible from the labels on the strings entering the square; this follows the convention of [2]. The number $\Delta_{n}$ will be defined by

$$
\Delta_{n}=(-1)^{n}\left(A^{2(n+1)}-A^{-2(n+1)}\right) /\left(A^{2}-A^{-2}\right),
$$

this being characterised by the Chebyschev recurrence relation

$$
\Delta_{n+1}=\Delta_{1} \Delta_{n}-\Delta_{n-1}
$$

where $\Delta_{1}=-A^{-2}-A^{2}$ and $\Delta_{0}=1$. The inductive defining formula for $f^{(n)}$ is shown in Figure $1, f^{(1)}$ being the identity in $T L_{1}$.

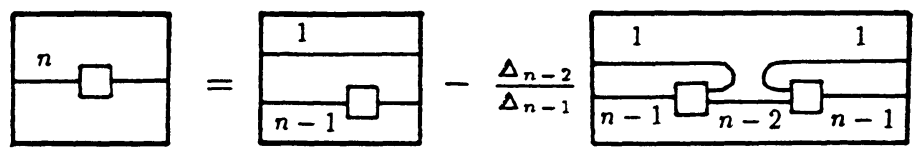

FIGURE 1

The formulae depicted in Figure 2 are immediately deducible (the third by induction on $i$ ).

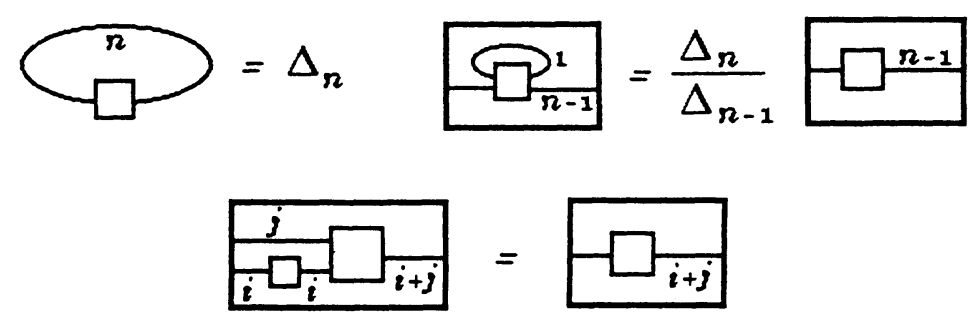

FIGURE 2

Note that when $A$ is chosen to be a primitive $4 r$ th root of unity, $\Delta_{n} \neq 0$ when $n \leq r-2, \Delta_{n}=0$ when $n=r-1$ and $f^{(n)}$ is not defined in $n \geq r$.

Consider the diagram shown in Figure 3. 


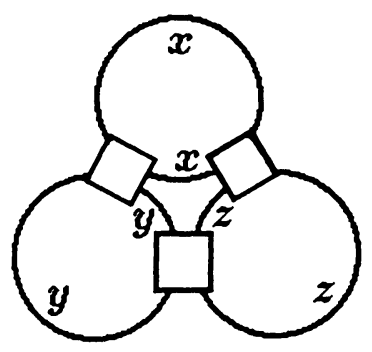

FIGURE 3

It consists of $x$ parallel copies of a circle, $y$ of another circle and $z$ of a third with $f^{(x+y)}, f^{(y+z)}$ and $f^{(z+x)}$ inserted as shown. Let $\Gamma(x, y, z)$ be the element of $\mathbb{S} S^{2}$ that this diagram represents. It will be important to know when $\Gamma(x, y, z)$ is and is not zero. This element is thus calculated in the following which serves also as an exercise in the use of the Temperley-Lieb algebra (the result of the lemma is inherent in [4] and implied in [2]; a simultaneously derived version of this proof appears in [9]). Here $\Delta_{n}$ ! denotes $\Delta_{n} \Delta_{n-1} \Delta_{n-2} \cdots \Delta_{1}$, this being interpreted as 1 if $n$ is -1 or zero.

\section{LEMMA 1.}

$$
\Gamma(x, y, z)=\left(\Delta_{x+y+z} ! \Delta_{x-1} ! \Delta_{y-1} ! \Delta_{z-1} !\right) /\left(\Delta_{y+z-1} ! \Delta_{z+x-1} ! \Delta_{x+y-1} !\right) .
$$

Proof. Consider the equations depicted in Figure 4. The first line follows from the defining relation in Figure 1 (together with $f^{(z)} e_{z-1}=$ 0 ), the second line follows by iterating the first line (and using the third equality of Figure 2).

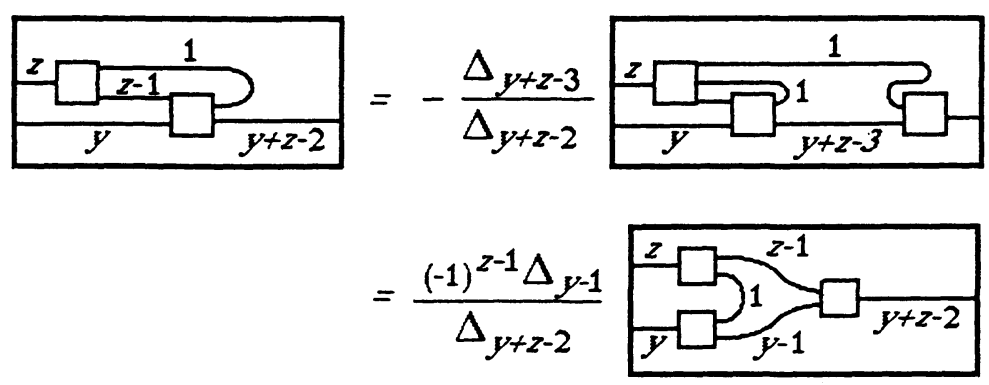

FIGURE 4

Next, the defining relation in Figure 1 followed by a double application of Figure 4 produces the identity of Figure 5. 


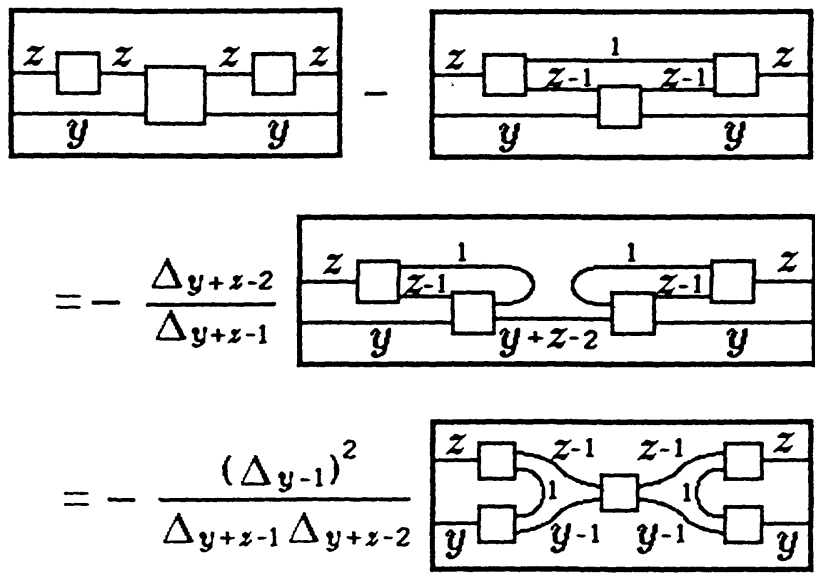

Figure 5

Now apply the identity of Figure 5 to the required object in Figure 3 using the second and third formula of Figure 2. The following recurrence relation results:

$$
\begin{aligned}
\Gamma(x, y, z)= & \Gamma(x, y, z-1) \Delta_{x+z} / \Delta_{x+z-1} \\
& -\Gamma(x+1, y-1, z-1)\left(\Delta_{y-1}\right)^{2} /\left(\Delta_{y+z-1} \Delta_{y+z-2}\right) .
\end{aligned}
$$

This is ready for a verification of the given formula by induction on $z$. That formula is clearly true when $z=0$ and inserting it into this recurrence relation reduces the proof to a demonstration of the equality

$$
\Delta_{x+y+z} \Delta_{z-1}=\Delta_{x+z} \Delta_{y+z-1}-\Delta_{y-1} \Delta_{x} .
$$

The truth of this can however easily be checked either directly from the formula for $\Delta_{n}$ or using a double induction on

$$
\Delta_{x+y}=\Delta_{x} \Delta_{y}-\Delta_{x-1} \Delta_{y-1} .
$$

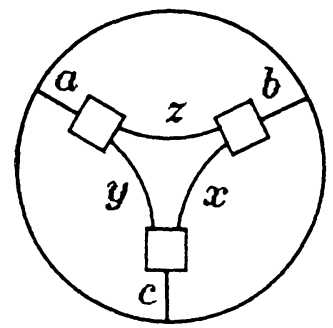

Figure 6

Consider the triad $T_{a, b, c}$ of Figure 6, introduced by Kauffman [2]. It is an element of $\mathbb{S} D$, the linear skein of the disc $D$, where now $D$ 
has an even number $a+b+c$ of specified boundary points partitioned into three subsets as shown. $T_{a, b, c}$ has the idempotents $f^{(a)}, f^{(b)}$, and $f^{(c)}$ placed just inside the disc adjacent to the three subsets. It is required that the integers $x, y$ and $z$ defined by $a=y+z, b=z+x$ and $c=x+y$ should all be non-negative. Suppose that $D^{\prime}$ is the disc complementary to $D$ in $S^{2}$ with the same specified boundary points. By means of the bilinear form $\mathbb{S} D \times \mathbb{S} D^{\prime} \rightarrow \mathbb{C}, T_{a, b, c}$ corresponds to the element $T_{a, b, c}^{*}$ of the dual of $\mathbb{S} D^{\prime}$.

Lemma 2. Let $A$ be a primitive $4 r$ th root of unity. Then $T_{a, b, c}^{*}$ is. non-zero if and only if $a+b+c \leq 2(r-2)$.

Proof. $\mathbb{S} D^{\prime}$ has a base consisting of all diagrams in $D^{\prime}$ with no crossing. However, for all but one of these diagrams there is an arc from a point of one of the three specified subsets (for example that with $a$ points) to another point of the same subset. The presence of the idempotent adjacent in $T_{a, b, c}$ to that subset ensures (using $\left.f^{(a)} e_{i}=0\right)$ that $T_{a, b, c}^{*}$ annihilates such an element. There remains to consider the base element of $\mathbb{S} D^{\prime}$ that consists of $z$ arcs from the first boundary subset to the second such subset, $x$ from the second to the third and $y$ from the third to the first. Of course $T_{a, b, c}^{*}$ maps this element to $\Gamma(x, y, z)$. It follows from Lemma 1 that, as $x+y+z$ increases, this is non-zero until $\Delta_{x+y+z} !=0$ and that occurs when $x+y+z=r-1$.

From now onwards fix $A$ as a primitive $4 r$ th root of unity.

Definition. A triple $(a, b, c)$ of non-negative integers will be called admissible if $a+b+c$ is even, $a+b+c \leq 2(r-2), a \leq b+c$, $b \leq c+a$ and $c \leq a+b$. This will be written $(a, b, c) \in \mathbb{A}$.

Note that the admissibility condition is just that a triple of nonnegative integers $(x, y, z)$ should exist as above and that $x+y+z \leq$ $r-2$. In such circumstances define $\Theta(a, b, c)=\Gamma(x, y, z)$.

3. Independence in 3-manifolds. Consider now the following situation. Let $M_{a}$ be a 3-manifold $M$ in $S^{3}$ having, as specified framed points in $\partial M, a$ points grouped in a small disc and $N$ other points. These $N$ points do not change in what follows. Let $M_{a}^{\prime}$ be the closure of the complement of $M_{a}$. Suppose that for each $a, 0 \leq a \leq r-2$, $\left\{X_{i, a}: i \in \mathfrak{I}(a)\right\}$ is a collection of elements of $\mathbb{S} M_{a}, \mathfrak{I}(a)$ being some indexing set, and that each $X_{i, a}$ has the idempotent $f^{(a)}$ placed adjacent to the $a$ points. Let $Y_{i, a, b, c}$ be obtained from $X_{i, a}$ by adding 
the triad $T_{a, b, c}$ as in Figure 7 (the 2-dimensional projections will be considered only in a neighbourhood of the $a$ points).

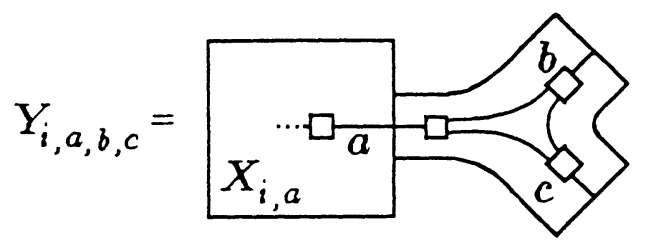

Figure 7

Lemma 3. Suppose that the $\left\{X_{i, a}^{*}: i \in \mathfrak{I}(a)\right\}$ are, for each $a$, independent elements of the dual of $\mathbb{S} M_{a}^{\prime}$. Then, for any $b$ and $c$, $\left\{Y_{i, a, b, c}^{*}:(a, b, c) \in \mathbb{A}, i \in \mathfrak{I}(a)\right\}$ are independent.

Proof. Suppose $\sum_{i, a} \lambda_{i, a} Y_{i, a, b, c}^{*}=0$. Then $\sum_{i, a} \lambda_{i, a} Z_{i, a, b, c, d}^{*}=$ 0 where $Z_{i, a, b, c, d}$ is as shown in Figure 8 . Note that in Figure 8, and in the remainder of the paper, a black dot is used, as in [2], to indicate the presence of a triad of the type of Figure 6.

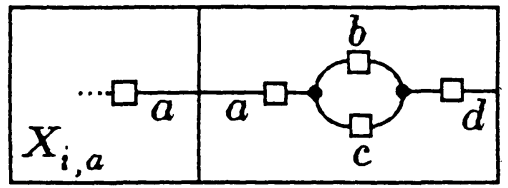

Figure 8

However it follows at once (see [2], for example) that

$$
Z_{i, a, b, c, d}^{*}=\delta_{a, d} \Theta(b, c, d) \Delta_{d}^{-1} X_{l, d}^{*} .
$$

So, letting $d=a$, for each $a$ such that $(a, b, c)$ is admissible,

$$
\sum_{i \in \mathfrak{I}(a)} \lambda_{i, a} \Theta(a, b, c) \Delta_{a}^{-1} X_{i, a}^{*}=0 .
$$

But the admissibility means that $\Theta(a, b, c) \neq 0$ (and $\Delta_{a} \neq 0$ ) so $\lambda_{i, a}=0$ for all $a$ and $i$.

COROLLARY. If triads are added together to form a tree

$$
X\left(a_{1}, a_{2}, \ldots, a_{s} ; i_{1}, i_{2}, \ldots, i_{s-3}\right)
$$

as in Figure 9, then, fixing $a_{1}, a_{2}, \ldots, a_{s}$ and allowing $i_{1}, i_{2}, \ldots, i_{s-3}$ to vary in all ways so that admissibility occurs at each node of the tree, the elements $\left\{X\left(a_{1}, a_{2}, \ldots, a_{s} ; i_{1}, i_{2}, \ldots, i_{s-3}\right)^{*}\right\}$ are independent. 


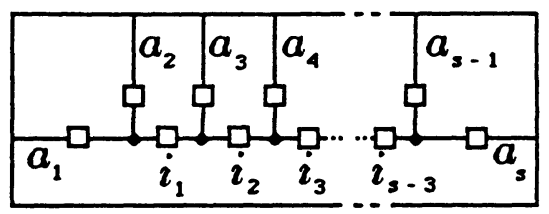

Figure 9

Suppose that an even number $a_{1}+a_{2}+\cdots+a_{s}$ of points are specified on the boundary of a disc $D$ the points grouped into $s$ subsets each containing $a_{i}$ points. Then, it is easy to see, in the following way, that the duals of all diagrams that have, for all $i$, the idempotent $f^{\left(a_{t}\right)}$ adjacent to the $i$ th subset, span the same subspace as $\left\{X\left(a_{1}, a_{2}, \ldots, a_{s} ; i_{1}, i_{2}, \ldots, i_{s-3}\right)^{*}\right\}$. This is because in the Temperley-Lieb algebra $T L_{n}, 1=f^{(n)}+R$ where $R$ is some sum of products of the $e_{i}$. As $f^{(r-1)^{*}}=0$ the dual of any diagram cutting a chord of the disc in more than $r-2$ points may be replaced by the sum of duals of diagrams cutting the chord fewer times. For $n \leq r-2$ the dual of a diagram whose arcs cross a given chord $n$ times may be replaced by a sum of duals of diagrams, in one of which the arcs cross the chord $n$ times but are then decorated with an $f^{(n)}$ and in the others the arcs cross fewer than $n$ times. At any stage an inadmissible triad can be neglected as its dual is the zero map. When $s=4$ this means there are two obvious bases for the same space. The situation is depicted in Figure 10 in which the diagrams represent elements of the dual space to $\mathbb{S} D^{\prime}$ when $D$ has $a+b+c+d$ specified points in $\partial D$. This is familiar from [2]; the summation is over all $i$ for which $(i, b, c)$ and $(i, a, d)$ are admissible. The elements $\left\{\begin{array}{lll}a & b & i \\ c & d & j\end{array}\right\}$ of this change of base matrix are referred to as (quantised) $6 j$-symbols.

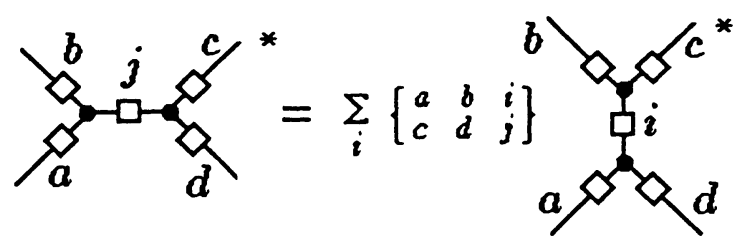

FIGURE 10

There now follows a somewhat technical little lemma that will prove useful.

LEMMA 4. Suppose that $(b, b, a)$ and $(a, c, c)$ are admissible triples. Then there is some $j, 0 \leq j \leq r-2$, such that the element of $\mathbb{S} S^{2}$ depicted by Figure 11 is non-zero. 


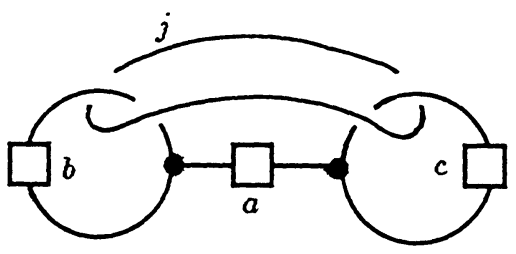

FIGURE 11

Proof. Note that the idempotent $f^{(j)}$ does not appear on the $j$ strands. Suppose that, to the contrary, the diagram represents zero for every $j$. Then, using the above-mentioned base change, the summation shown in Figure 12 is zero.

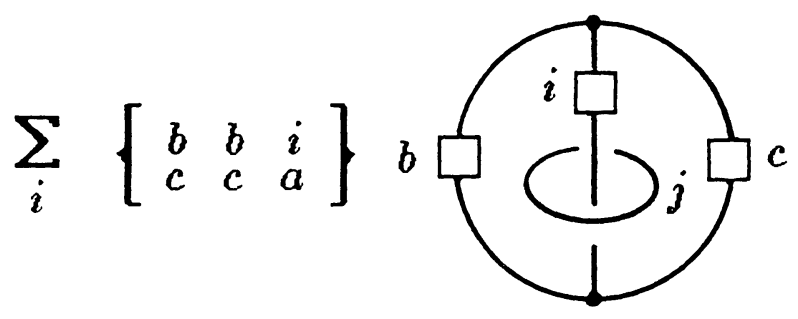

FIGURE 12

However, by Lemma 6 of [7], this means that

$$
\sum_{i}\left\{\begin{array}{lll}
b & b & i \\
c & c & a
\end{array}\right\}\left(\alpha_{i}\right)^{j} \Theta(b, i, c)=0,
$$

where $\alpha_{i}=-A^{2(i+1)}-A^{-2(i+1)}$. Because $A$ is a primitive $4 r$ th root of unity $\alpha_{0}, \alpha_{1}, \ldots, \alpha_{r-2}$ are all distinct. Thus the Vandermonde matrix $\left(\alpha_{i}\right)^{j}$ is non-singular, and so there can be no non-trivial linear relation between its rows (compare [7]). Thus

$$
\left\{\begin{array}{lll}
b & b & i \\
c & c & a
\end{array}\right\} \Theta(b, i, c)=0
$$

for all $i$ for which $(b, i, c)$ is admissible. But then $\Theta(b, i, c) \neq 0$ so that $\left\{\begin{array}{lll}b & b & i \\ c & c & a\end{array}\right\}$ is zero for all such $i$. That however means that a whole row of a change of base matrix is zero, a contradiction.

Note that it follows that the lemma is equally true if the idempotent $f^{(j)}$ is now inserted on the $j$ strands.

Corollary. Let $U_{a, b, j}$ be the element of $\mathbb{S} D$ in Figure 13. Then $U_{a, b, j}^{*}=\mu_{a, b, j} T_{a, j, j}^{*}$, and for each $a$ and $b$ with $(a, b, b)$ 
admissible, there exists $a j$, with $0 \leq j \leq r-2$ and $(a, j, j)$ admissible, such that $\mu_{a, b, j} \neq 0$.

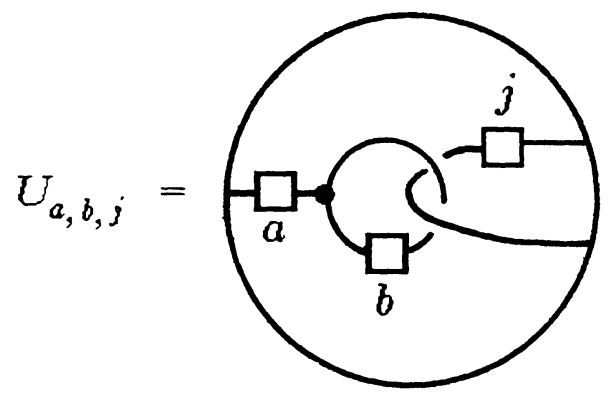

FIGURE 13

Proof. $U_{a, b, j}^{*}$ and $T_{a, j, j}^{*}$ both annihilate all but the same single base element of $\mathbb{S} D^{\prime}$. Thus they differ by multiplication by a scalar $\mu_{a, b, j}$. However by Lemma 4 , for each $a$ and $b$, with $(a, b, b)$ admissible, there is some $j$ for which $U_{a, b, j}^{*}$ is not the zero map.

Return now to the notation at the beginning of this section in which $\left\{X_{i, a}: i \in \mathfrak{I}(a)\right\}$ is a collection of elements of $\mathbb{S} M_{a}$ each having the idempotent $f^{(a)}$ placed adjacent to the set of $a$ points in $\partial M$. Suppose that $W_{i, a, b}$ is as shown in Figure 14.

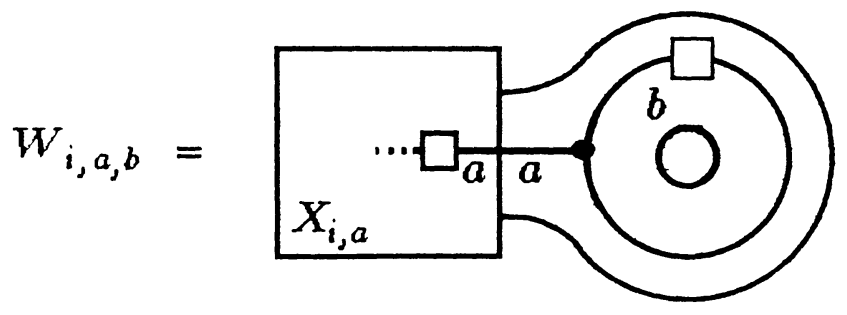

FIGURE 14

Note that $W_{i, a, b}$ is in the linear skein of a new 3-manifold in $S^{3}$ obtained by adding a 1 -handle to $M$ in a trivial manner.

LEMma 5. Suppose that the $\left\{X_{i, a}^{*}: i \in \mathfrak{I}(a)\right\}$ are, for each $a$, independent elements of the dual of $\mathbb{S} M_{a}^{\prime}$. Then $\left\{W_{i, a, b}^{*}:(a, b, b) \in\right.$ $\mathbb{A}, i \in \mathfrak{I}(a)\}$ are independent.

Proof. Suppose that $\sum_{i, a, b} \lambda_{i, a, b} W_{i, a, b}^{*}=0$, the sum being over all $(i, a, b)$ such that $0 \leq a \leq r-2, i \in \mathfrak{I}(a)$ and $(a, b, b)$ is 
admissible. Then, for all $j$ and $k$,

$$
\sum_{i, a, b} \lambda_{i, a, b} W_{i, a, b, j, k}^{+*}=0
$$

where $W_{i, a, b, j, k}^{+}$is shown in Figure 15 .

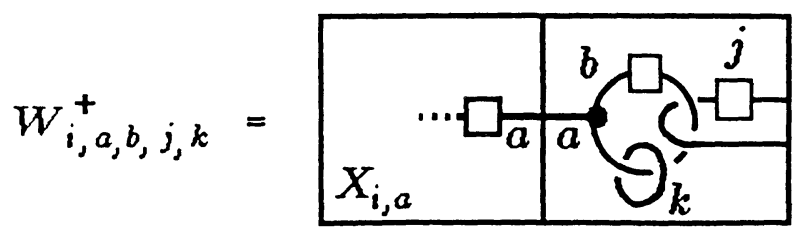

FIGURE 15

Then

$$
\sum_{i, a, b} \lambda_{i, a, b}\left(\alpha_{b}\right)^{k}\left(X_{i, a} \cup U_{a, b, j}\right)^{*}=0
$$

where, as before, the $\left(\alpha_{b}\right)^{k}$ are the elements of a non-singular Vandermonde matrix. Hence, for each $b$,

$$
\sum_{i, a} \lambda_{i, a, b}\left(X_{i, a} \cup U_{a, b, j}\right)^{*}=0
$$

By the corollary to Lemma 4 , for each $b$

$$
\sum_{i, a} \lambda_{i, a, b}\left(X_{i, a} \cup \mu_{a, b, j} T_{a, j, j}\right)^{*}=0 .
$$

Then, by Lemma 3 , if $(a, j, j)$ is admissible $\lambda_{i, a, b} \mu_{a, b, j}=0$. But the corollary to Lemma 4 states that, for each $a$ and $b$, such a $j$ can be selected so that $\mu_{a, b, j} \neq 0$. Hence $\lambda_{i, a, b}=0$ for all $(a, b, i)$ for which $i \in \mathfrak{I}(a)$ and $(a, b, b)$ is admissible.

THEOREM. Suppose that $M$ is a handlebody in $S^{3}$ (with no specified points in its boundary), and that $M^{\prime}$, the closure of its complement, is also a handlebody. Let $A$ be a primitive 4 rth root of unity. $A$ base for the quotient of $j M$ by the kernel of the natural map $\mathbb{S} M \rightarrow$ $\operatorname{Hom}\left(\mathbb{S} M^{\prime}, \mathbb{C}\right)$ is as follows. Project $M$ to a disc with holes. The duals of all diagrams as shown in Figure 16 that have an admissible triad at each node form the required base. 


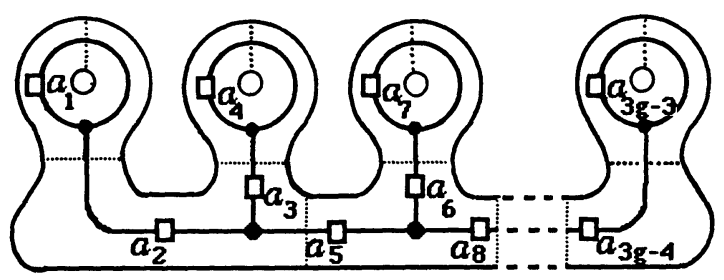

FIGURE 16

Proof. That the given set spans the quotient follows in a way similar to the remarks following Lemma 3: The infinite spanning set consisting of the duals of all diagrams with no crossing and no null-homotopic closed curve can, as there explained, be reduced to a finite set in which no diagram cuts any of the dotted chords of Figure 16 in more than $r-2$ points, and the Temperley-Lieb idempotents can likewise be inserted where the diagrams cross those chords. The only remaining diagrams with non-zero duals are those consisting of admissible triads between the dotted chords. Independence is an immediate consequence of Lemmas 3 and 5.

Note 1 . Other bases can be obtained by modifying the above base at two adjacent nodes of Figure 16, using the $6 j$-symbols as the change of base matrix as in Figure 10.

Note 2. The idempotents can be removed from all the triads of Figure 16 and the result is still a base (the result is clearly still a spanning set and it has the same number of elements as has the base).

Note 3. If $M$ is a ball the dimension of the quotient space is 1 . If $M$ is a solid torus the dimension is $r-1$ and if $M$ has genus 2 the dimension is $\left(r^{3}-r\right) / 6$.

Note 4. If one begins with a closed connected orientable surface and specifies in it a collection of simple closed curves that separate the surface into copies of a disc with two holes ('pairs of pants'), spanning: those curves with discs, then adding 3-balls, produces a handlebody bounded by the surface. The discs give a decomposition of the handlebody into triad-like pieces ready for the construction of a base of the relevant (quotient) vector space as above. 


\section{REFERENCES}

[1] M. F. Atiyah, The Geometry of Physics and Knots, Cambridge Univ. Press, (1990).

[2] L. H. Kauffman, Knots and Physics, World Scientific, (1991).

[3] L. H. Kauffman and S. Lins, A 3-manifold invariant by state summation, preprint, University of Illinois at Chicago, (1991).

[4] A. N. Kirillov and N. Yu. Reshetikhin, Representations of the algebra $U_{q}(\operatorname{Sl}(2))$, $q$-orthogonal polynomials and invariants of links. Infinite dimensional Lie algebras and groups, ed. V. G. Kac, World Scientific, (1989).

[5] W. B. R. Lickorish, Linear skein theory and link polynomials, Topology and its Appl., 27 (1987), 265-274.

[6] _ Three-manifold invariants from the combinatorics of the Jones polynomial, Pacific J. Math., 149 (1991), 337-347.

[7] - Three-manifolds and the Temperley-Lieb algebra, Math. Ann., 290 (1991), 657-670.

[8] _ Calculations with the Temperley-Lieb algebra, Comment. Math. Helv., 67 (1992), 571-591.

[9] G. Masbaum and P. Vogel, 3-valent graphs and the Kauffman bracket, preprint, Université Paris VII, (December 1991).

[10] J. H. Przytycki, Skein modules of 3-manifolds, Bull. Pol. Acad. Sci. Math., (to appear).

[11] P. Vogel, Seminar at the Universite de Provence, Marseille, (April 1991).

[12] E. Witten, Quantum field theory and Jones' polynomial, Comm. Math. Phys., 121 (1989), 351-399.

Received January 2, 1992.

UNIVERSITY OF CAMBRIDGE

CAMbridge, CB2 1SB, ENGland 



\title{
PACIFIC JOURNAL OF MATHEMATICS \\ Founded by \\ E. F. BecKenbaCH (1906-1982) F. Wolf (1904-1989)
}

\section{EDITORS}

V. S. VARADARAJAN
(Managing Editor)
University of California
Los Angeles, CA 90024-1555
vsv@math.ucla.edu
F. MICHAEL CHRIsT
University of California
Los Angeles, CA 90024-1555
christ@math.ucla.edu
HerBeRT CLEMENs
University of Utah
Salt Lake City, UT 84112
clemens@math.utah.edu

V. S. VARADARAJAN University of California Los Angeles, CA 90024-1555 @math.ucla.edu Los Angeles, CA 90024-1555

Herbert Clemens clemens@math.utah.edu

\author{
THOMAS ENRIGHT \\ University of California, San Diego \\ La Jolla, CA 92093 \\ tenright@ucsd.edu \\ Nicholas ERcolani \\ University of Arizona \\ Tucson, AZ 85721 \\ ercolani@math.arizona.edu \\ R. FINN \\ Stanford University \\ Stanford, CA 94305 \\ finn@gauss.stanford.edu \\ VAUGHAN F. R. JoNES \\ University of California \\ Berkeley, CA 94720 \\ vfr@math.berkeley.edu
}

\author{
STEVEN KeRCKHOFF \\ Stanford University \\ Stanford, CA 94305 \\ spk@gauss.stanford.edu \\ MarTin SCHARLEMANN \\ University of California \\ Santa Barbara, CA 93106 \\ mgscharl@henri.ucsb.edu \\ HAROld STARK \\ University of California, San Diego \\ La Jolla, CA 92093
}

\section{SUPPORTING INSTITUTIONS}

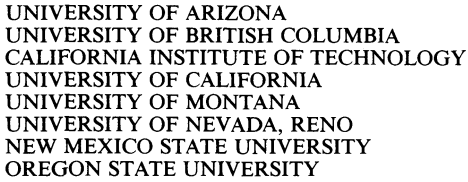

The Supporting Institutions listed above contribute to the cost of publication of this Journal, but they are not owners or publishers and have no responsibility for its content or policies.

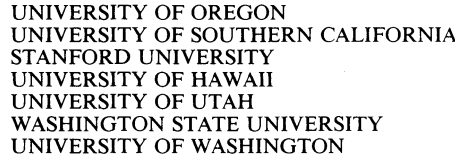

\begin{abstract}
Mathematical papers intended for publication in the Pacific Journal of Mathematics should be in typed form or offset-reproduced (not dittoed), double spaced with large margins. Please do not use built up fractions in the text of the manuscript. However, you may use them in the displayed equations. Underline Greek letters in red, German in green, and script in blue. The first paragraph must be capable of being used separately as a synopsis of the entire paper. In particular it should contain no bibliographic references. Please propose a heading for the odd numbered pages of less than 35 characters. Manuscripts, in triplicate, may be sent to any one of the editors. Please classify according to the 1991 Mathematics Subject Classification scheme which can be found in the December index volumes of Mathematical Reviews. Supply name and address of author to whom proofs should be sent. All other communications should be addressed to the managing editor, or Julie Speckart, University of California, Los Angeles, California 90024-1555.

There are page-charges associated with articles appearing in the Pacific Journal of Mathematics. These charges are expected to be paid by the author's University, Government Agency or Company. If the author or authors do not have access to such Institutional support these charges are waived. Single authors will receive 75 free reprints; joint authors will receive a total of 100 free reprints. Additional copies may be obtained at cost in multiples of 50 .
\end{abstract}

The Pacific Journal of Mathematics (ISSN 0030-8730) is published monthly except for July and August. Regular subscription rate: $\$ 200.00$ a year (10 issues). Special rate: $\$ 100.00$ a year to individual members of supporting institutions.

Subscriptions, orders for numbers issued in the last three calendar years, and changes of address should be sent to Pacific Journal of Mathematics, P.O. Box 969, Carmel Valley, CA 93924, U.S.A. Old back numbers obtainable from Kraus Periodicals Co., Route 100, Millwood, NY 10546.

The Pacific Journal of Mathematics at P.O. Box 969, Carmel Valley, CA 93924 (ISSN 0030-8730) is published monthly except for July and August. Second-class postage paid at Carmel Valley, Califorñia 93924, and additional mailing offices. Postmaster: send address changes to Pacific Journal of Mathematics, P.O. Box 969, Carmel Valley, CA 93924.

\section{PUBLISHED BY PACIFIC JOURNAL OF MATHEMATICS, A NON-PROFIT CORPORATION}

This publication was typeset using $\mathcal{A} \mathcal{M S}-\mathrm{T}_{\mathrm{E}} \mathrm{X}$,

the American Mathematical Society's $T_{E} X$ macro system.

Copyright (C) 1993 by Pacific Journal of Mathematics 


\section{PACIFIC JOURNAL OF MATHEMATICS}

Volume $159 \quad$ No. $2 \quad$ June 1993

$L^{p}$-integrability of the second order derivatives of Green potentials in 201 convex domains

VilHeLM AdOLFSSON

Solutions of the stationary and nonstationary Navier-Stokes equations 227 in exterior domains

ZHi MiN CHEN

Mesures de Patterson-Sullivan sur le bord d'un espace hyperbolique au 241 sens de Gromov

Michel COORNAERT

Differential-difference operators and monodromy representations of 271 Hecke algebras

CHARLES F. DUNKL

Between the unitary and similarity orbits of normal operators

PAUl GuinAND and LAURENT WALSh MARCOUX

Skeins and handlebodies

W. B. RAYMOND LICKORISH

The Plancherel formula for homogeneous spaces with polynomial spectrum

RONALD LESLIE LIPSMAN

On the uniform approximation problem for the square of the

Cauchy-Riemann operator

JoAn Manuel Verdera Melenchón 